\title{
El futuro del transporte en Costa Rica: transición al vehículo eléctrico y otras tecnologías
}

\section{The future of transport in Costa Rica: transition to electric vehicles and other technologies}

\author{
José Vega Córdoba* \\ *Universidad Técnica Nacional, Costa Rica. Contacto: jovegaca@est.utn.ac.cr
}

Referencia/ reference:

Vega, C. (2020). El futuro del transporte en Costa Rica: transición al vehículo eléctrico y otras tecnologías. Yulök Revista de Innovación Académica, 4(1), 10-20

\section{Resumen}

En este estudio de caso se realizará un análisis de las condiciones técnicas que debe cumplir Costa Rica para la electrificación del transporte. El país reúne muchas de estas condiciones, generación de electricidad renovable, políticas y planes de transporte eléctrico, empresas distribuidoras interesadas y otros factores positivos. Sin embargo, se analizarán los problemas actuales principales como, la dependencia de hidrocarburos, los desafíos a superar para alcanzar transición correcta, el efecto sobre el Sistema Eléctrico Nacional, distribución de estaciones de carga rápida y la integración de los vehículos eléctricos en redes inteligentes.

Palabras clave: energía renovable, vehículos eléctricos, transporte, emisiones, tecnología.

\section{Abstract}

In this case study, an analysis was made about the technical conditions that Costa Rica must meet for the transport electrification. The country meets many of these requirements, renewable electricity generation, electric transport policies and plans, interested utilities and other positive factors. However, the main current problems like, the dependence on fossil fuel, the challenges to overcome in order to achieve the correct transition, the effect on the National Electric System, distribution of fast-charging stations and the integration of electric vehicles into smart grids will be analyzed.

Keywords: renewable energy, electric vehicles, transport, emissions, technology. 


\section{Introducción}

Durante los últimos años, se han investigado y desarrollado nuevas tecnologías con el objetivo de mejorar los métodos de transporte vehicular. Los vehículos eléctricos (VE) incorporan dichas tecnologías y logran alcanzar una mayor eficiencia del uso de energía en comparación con los vehículos convencionales que poseen un motor de combustible basado en hidrocarburos. Actualmente, el vehículo eléctrico supone una transición en las sociedades en desarrollo y está llegando al punto de disrupción donde el costo-beneficio es llamativo para los consumidores. Sin embargo, más allá del beneficio monetario trae consigo una serie de ventajas, en las que se destaca la ausencia de emisiones de gases contaminantes.

Costa Rica tiene el objetivo de alcanzar un transporte de cero emisiones y crear un sistema energético sustentable y de bajas emisiones de gases de efecto invernadero (GEI). Según el balance energético acumulado a abril del 2020 brindado por el informe mensual del Centro Nacional de Control de Energía (2020) el país ha generado el 99,38 $\%$ de su electricidad con fuentes de energía renovables, por lo tanto se ve obligado a reducir emisiones en otros sectores como el transporte. En el balance energético nacional registrado en el 2018, la mayor parte de energía es consumida en transporte, el cual representa aproximadamente el $61 \%$ del consumo total de energía secundaria, correspondiendo cerca del $28 \%$ al transporte privado (Secretaría de Planificación del Subsector Energía, 2019).
Por consiguiente, se han establecido en la legislación varios incentivos para los compradores de vehículos eléctricos tales como exoneración de impuestos de importación, exoneración gradual de marchamo en los primeros cinco años, privilegios en algunos parqueos, entre otros.

Aun considerando que Costa Rica tiene un panorama favorable para la transición, ¿está la red eléctrica actual preparada para la electrificación del transporte? ¿Cuáles son los retos a los que se enfrentará el país en el proceso? ¿Cuáles beneficios económicos trae al consumidor? ¿Qué estructuras eléctricas se requieren para la electrificación? El objetivo de este estudio es responder a estas interrogantes y analizar los desafíos técnicos y económicos que se deben superar para alcanzar una electrificación madura del parque vehicular costarricense. Además de estudiar las nuevas tecnologías requeridas para alcanzar la transformación.

\section{Actual dependencia de hidrocarburos}

Costa Rica no ha podido resolver la dependencia de los hidrocarburos como fuente de energía, la causa principal es el incremento de automóviles particulares y motocicletas, cuya tecnología se basa en motores de combustión interna. El parque vehicular creció un 3,4\% entre 2017 y 2018, registrando 1711834 de unidades (Programa Estado de la Nación, 2019). Este aumento de vehículos puede estar ligado a la ineficiencia del transporte público, en este contexto la calidad del servicio, la inseguridad

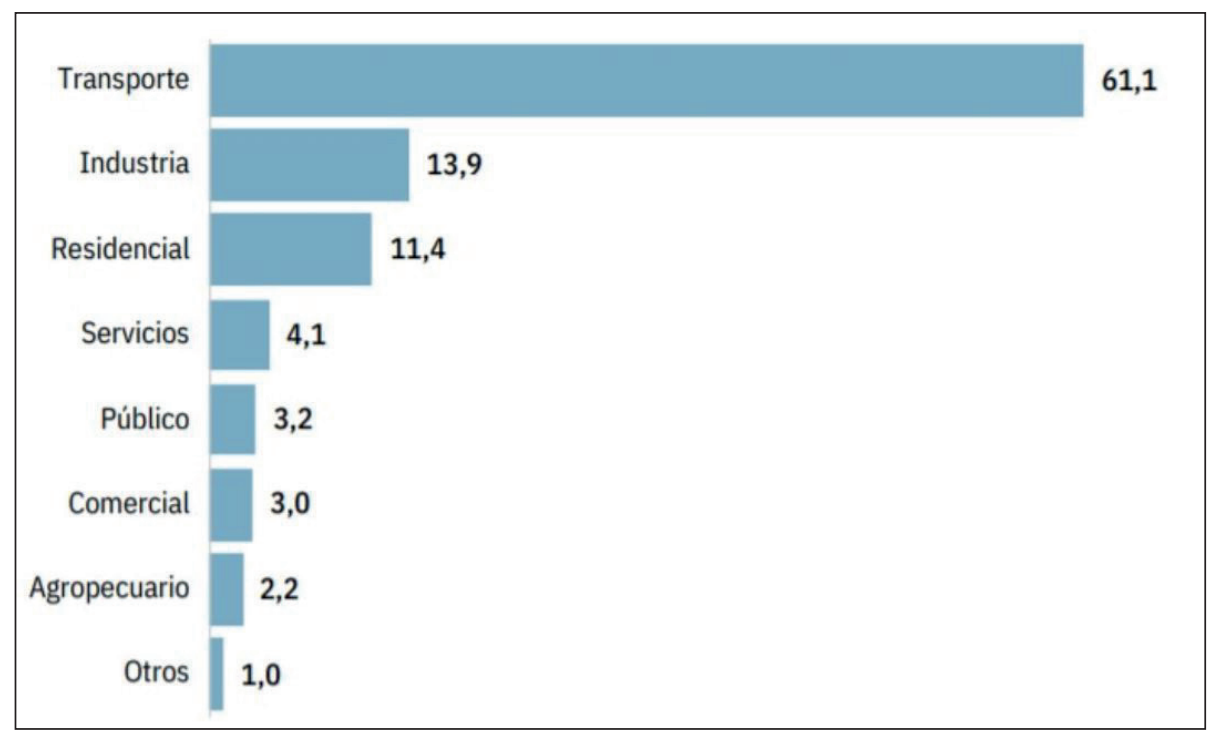

Figura 1. Distribución del consumo en porcentaje de energía secundaria, por sector, en el 2018. Fuente: Datos del Balance de Armonía con la naturaleza, y Sepse-Minae, 2019 (Informe Estado Nación, 2019, p. 57). 
y horarios limitados, son algunas razones por las que se prefiere el transporte privado (Arrieta, 2018).

Lo anterior se refleja en los indicadores de consumo, según datos de la Secretaría de Planificación del Subsector Energía en 2018 la demanda fue abastecida, mayoritariamente, por derivados del petróleo $(73,5 \%)$, electricidad $(24,1 \%)$ y el coque. El consumo se concentró en transporte, industria y residencial, que sumados representan más del $85 \%$ del total de energía (Programa Estado de la Nación, 2019). Es importante destacar que, en los acuerdos de París, Costa Rica se comprometió a reducir la emisión GEI de 12,4 millones de toneladas de $\mathrm{CO}_{2}$ del año 2012 a 9,4 millones al 2030 siguiendo con el programa de carbono neutralidad. (figura 1 )

Como se observa en la figura 1 es en el sector transporte donde los costarricenses consumen más energía. Según Arrieta (2018) para el 2017 se registraba una densidad de 231 carros por cada 1000 habitantes, la tercera más alta de Latinoamérica solo superada por México y Argentina. Además, la mayoría de los autos tienen más de 16 años de antigüedad lo que aumenta el impacto ambiental. Esta gran cantidad de vehículos no solo agudiza los problemas de ambiente, sino también de salud, puesto que al existir más vehículos la expulsión de gases aumenta y la calidad del aire disminuye, esto es lo que produce, en algunos casos, problemas de salud. Esto aunado al congestionamiento vehicular en zonas urbanas, que evidentemente empeora año con año.

Asimismo, en el país se estudia el proyecto de Ley N. ${ }^{\circ}$ 20227 sobre la Transición al Transporte no Contaminante, donde se procura establecer al año 2030 como límite para la importación de autos con motor de combustión interna (Umaña, 2017), lo cual establece una reestructuración de la flotilla a vehículos impulsados por otras tecnologías más limpias y eficientes.

\section{Vehículos eléctricos}

Los vehículos eléctricos vienen a transformar el transporte en general, incluso con respecto al combustible, las emisiones de carbono, costos, reparaciones y hábitos de manejo. El impulso actual más importante es la descarbonización para contribuir a la solución de la emergencia del cambio climático. No obstante, según Crabtree (2019) se espera que pronto puedan provocar un cambio en la economía, ya que se anticipa una disminución del costo de fabricación de la producción de estos y por ende serán más baratos y eficientes que sus homólogos de gasolina o diésel. El mejor exponente de electrificación de su flotilla vehicular es Noruega, quienes apuntan a que el $100 \%$ de sus automóviles sean eléctricos e híbridos enchufables para el año 2025 (Crabtree, 2019). Cabe señalar que este país tiene una cantidad de población similar a la de Costa Rica y también producen su energía eléctrica, mayoritariamente, con fuentes renovables, sin embargo, su economía es superior.

Costa Rica por sus recursos energéticos tiene un futuro favorable para cambiar su flotilla vehicular a eléctrica, ya que la red es alimentada en un $99 \%$ por fuentes renovables, es estable y se cubre prácticamente el $99 \%$ de la población (Umaña, 2017). En este escenario, utilizar la potencia eléctrica en el transporte tiene una marca significativa al sustituir los combustibles fósiles por electricidad generada a partir de fuentes renovables.

En los últimos diez años hasta primer semestre del 2019 se han importado al país 714 carros $100 \%$ eléctricos, desde el 2009 hasta el primer semestre de 2019, esto coloca a Costa Rica dentro de los países de Latinoamérica con más automóviles de esta tecnología, solo detrás de Colombia y México (Herrera, 2019).

\section{Cargadores eléctricos y electrolineras}

El país debe estar preparado para empezar la difusión del uso de este tipo de vehículos, y uno de los requisitos más importantes es la proliferación de las estaciones de carga. Los niveles de recarga se clasifican de acuerdo con la potencia consumida y tiempo de recarga. En Estados Unidos se utiliza el estándar SAE J1772 y en Europa las normas IEC-61851-1, IEC 62196-2. Cada estándar tiene diferentes modalidades de operación tanto en CA como en $\mathrm{CC}$ en donde se varía principalmente la potencia a la que puede cargarse el vehículo (Brenes, 2016).

En cuanto a la estandarización de un conector universal para carga rápida siguen existiendo diferencias entre fabricantes. En Norteamérica y Europa se respalda el CCS (en inglés, Combined Charging System), mientras que Japón y sus fabricantes usan CHAdeMO y China, que tiene los mayores usos del mercado de vehículos eléctricos del mundo GB/T (Enel X, 2019). Además, existe el Supercharger de Tesla, que es un sistema patentado por Tesla Motors. En Costa Rica las estaciones de recarga rápida 
suelen disponer de dos cargadores uno CSS (cubre la mayoría de los fabricantes) y CHAdeMO (para algunos modelos de Nissan y Mitsubishi) (Plugshare, 2019).

A continuación, se definen algunas clasificaciones de los niveles de las estaciones de recarga.

Estaciones de carga de nivel 1 (Cable del carro): este tipo de cargadores puede ser utilizados en los hogares en una salida de tomacorriente común de $120 \mathrm{~V}$ AC. Es la opción más económica, no obstante, es la que brinda la recarga más lenta. Normalmente, los fabricantes incluyen estas estaciones en el vehículo para que los propietarios puedan cargarlos durante la noche en sus hogares.

Estaciones de carga de nivel 2 (Semirápido): estos a diferencia de los cargadores de nivel 1 no pueden conectarse a una salida convencional, sino que deben ser instalados por técnicos en electricidad. Se conectan a un circuito dedicado de 240V AC. Dependiendo de la corriente de salida pueden cargar el vehículo entre hora y media a dos horas.

Estaciones de carga de nivel 3 (Cargadores rápidos): estas estaciones pueden proveer $100 \mathrm{~km}$ de autonomía en solo 20 minutos, dependiendo del fabricante y modelo de vehículo. No todos los equipos pueden ser recargados con esta tecnología.

En Costa Rica se cuenta con varios cargadores instalados sobre el territorio, la mayoría son con salida del tipo J1772, tal como se ve en el resumen de la figura 2.
Aunque la mayoría de la carga de los vehículos eléctricos se realice en los hogares o en lugares de trabajo, garantizar que los conductores siempre puedan llegar a una estación de carga reduciría la ansiedad de quedarse sin energía en la batería, particularmente cuando se viaja una distancia por encima de la autonomía del vehículo eléctrico, que actualmente es de unos $200 \mathrm{~km}$ (Gallardo, et al., 2019). Esta "ansiedad por el rango" o "ansiedad por la autonomía" se define como la preocupación del usuario del VE de quedarse sin energía (Faraj y Basir, 2016), para evitar esto se debe planificar la ruta con anticipación de tal manera que el conductor tenga asegurado el acceso a la recarga de energía. Por lo tanto, una red de estaciones de carga se convierte en un activo necesario para empezar a adoptar el vehículo eléctrico. Estas estaciones suelen estar compuestas de cargadores de nivel 2 (rango de potencias entre $3,7 \mathrm{~kW}$ hasta $22 \mathrm{~kW}$ ) o nivel 3 (desde $22 \mathrm{~kW}$ hasta $200 \mathrm{~kW}$ ). El tiempo de carga varía, proporcionalmente, a la potencia entregada. Por un lado, un cargador rápido de $50 \mathrm{~kW}$ puede tardar, aproximadamente, 30 minutos en cargar el vehículo eléctrico al $80 \%$ de una capacidad de batería de $28 \mathrm{kWh}$. Por otro lado, los cargadores más lentos pueden tomar de 1 a 2 horas para la misma capacidad. (tabla 1)

El tiempo de carga aumenta proporcionalmente a la capacidad de la batería, mientras que disminuye para una mayor potencia de los cargadores. La rapidez de las estaciones de servicio puede atraer a los compradores potenciales de vehículos eléctricos que están acostumbrados a los autos de combustión interna, para quienes los cargadores de nivel 3 (rápidos), también conocidos como estaciones de carga rápida, serían más convenientes (menores tiem-

\section{Distribución de los conectores}

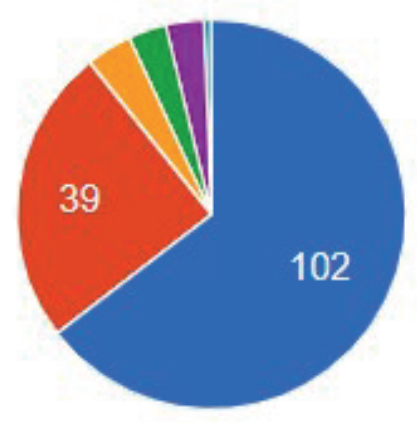

TYPE 1 (SAE J1772)

NEMA 5-15 (US Plug)

Type I (AU, NZ, CN Plug)

CCS2

CHAdeMO

Tesla Dest.Charger

Figura 2. Distribución de conectores no oficial en el territorio nacional, estadísticas recolectadas al 12 de diciembre de 2019. Fuente: Puntos de recarga Costa Rica (Electromaps, 2019). 
Tabla 1. Comparación de tensión y corriente de salida, tiempo de recarga y costo de diferentes tipos de cargadores de vehículos eléctricos existentes

\begin{tabular}{lccc|}
\hline & $\begin{array}{c}\text { Suministro de } \\
\text { electricidad }\end{array}$ & $\begin{array}{c}\text { Tiempo para recargar } \\
\text { uso diario típico }\end{array}$ & $\begin{array}{c}\text { Costo incl. } \\
\text { instalación** }\end{array}$ \\
\hline Cargador rápido & 240V - hasta 80A & 20 minutos* & $\$ 1.500-3.000$ \\
\hline Cargador semi-rápido & $240 \mathrm{~V}-16-40 \mathrm{~A}$ & $35-90$ minutos* & $\$ 1.000-2.000$ \\
\hline Cable del carro & $120 \mathrm{~V}-10 \mathrm{~A}$ & $3-5$ horas & Gratis \\
\hline & $\begin{array}{l}\text { *Depende de la potencia del cargador de a bordo del carro } \\
\text { **Depende de la necesidad de instalar circuitos nuevos y reforzar circuitos existentes }\end{array}$ \\
\hline
\end{tabular}

Fuente: Escoia y Costa Rica Limpia (Utgård, Bermúdez y Araya, 2016, p. 8)

pos de espera). A pesar de esto, los beneficios de invertir en infraestructura de carga son inciertos y se desconoce la proporción de nuevos vehículos eléctricos para cada nueva estación de carga, aunque más de un punto de carga por cada 10 vehículos eléctricos puede resultar costoso e ineficaz (Gallardo et al., 2019).
En Costa Rica los esfuerzos por parte de las compañías distribuidoras de electricidad son evidentes. Se han instalado diferentes tipos de cargadores a lo largo del territorio nacional y según la búsqueda en Plugshare (2019) la mayoría son de nivel 2 (L2) es decir, semirápidos. Además, es necesario aclarar que esta aplicación tiene información suministrada por usuarios propietarios de carros eléctricos, por lo tanto, podrían faltar puntos de recarga o quizás repetirse por error de registro.

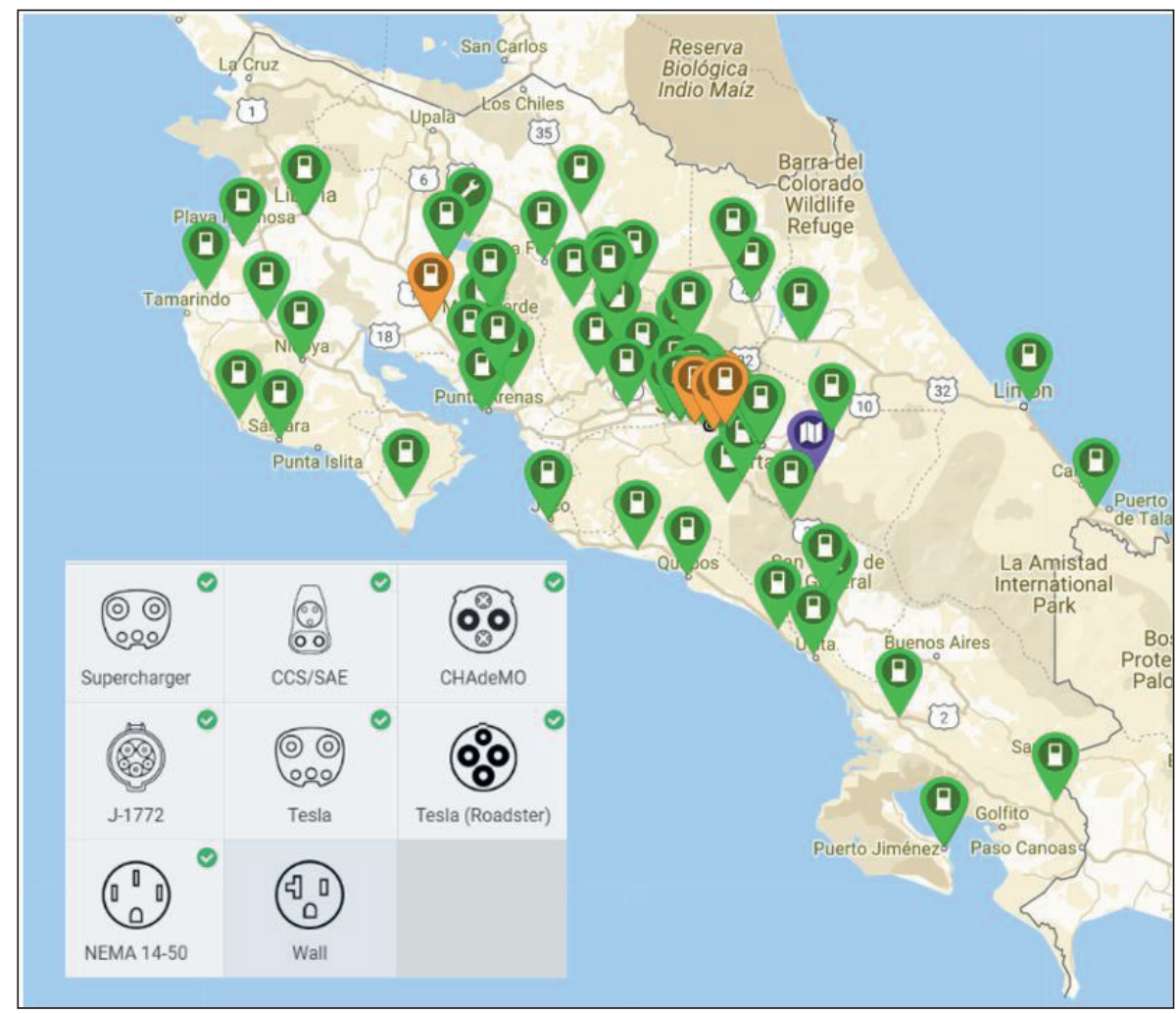

Figura 3. Distribución de estaciones no oficiales de carga en Costa Rica y tipos de conectores de vehículos eléctricos. Fuente: figura obtenida mediante aplicación Plugshare (Plugshare, 2019). 
Debido a que en el país la mayoría de las familias poseen un estacionamiento propio dentro del hogar y con acceso a electricidad, esto no supone un problema para el abastecimiento de energía del vehículo, sin embargo, es necesario contar con estaciones de carga rápida para impulsar aún más la adaptación. El Gobierno ha anunciado en el 2019 que se instalarán 34 cargadores rápidos con el apoyo del Instituto Costarricense de Electricidad y las demás compañías de energía. Según el comunicado se contará con una plataforma informática para poder reservar y pagar por el uso de la estación de recarga. (Presidencia de República de Costa Rica, 2019). Las compañías de servicios eléctricos son quienes deciden la distribución de los cargadores y en este contexto se firmó el Decreto N. ${ }^{\circ}$ 41642-MINAE para la construcción y el funcionamiento de la red que establece la ubicación futura de los centros de carga.

\section{Beneficios económicos}

Más allá de las cero emisiones de estos equipos y las nuevas tecnologías que incorporan, el consumidor busca beneficio. Lo cierto es que los autos eléctricos en Costa Rica tienen un precio aproximado de USD30.000, con exoneraciones aplicadas y es aquí donde los consumidores se preguntan ¿Es más barato utilizar combustibles fósiles o electricidad? Al tomar en consideración que en Costa Rica se conducen en promedio $57 \mathrm{~km}$ diarios; con vehículos de $200 \mathrm{~km}$ de autonomía hay que recargarlos cada tres días. Los usuarios costarricenses recorren cerca de 20 000 o $21000 \mathrm{~km}$ anuales según estadísticas nacionales, lo que equivale a $1750 \mathrm{~km}$ por mes, esto tiene un costo de USD150 de combustible tipo gasolina súper contra USD50 dólares de electricidad recorriendo la misma distancia y según los precios del petróleo y tarifas eléctricas para junio del 2019 (Quirós y Blanco, 2019). Esto quiere decir que, actualmente, en el país es tres veces más barato utilizar los autos eléctricos que los de combustión solo en el rubro de operación. Ahora, si más vehículos ingresan al país las tarifas eléctricas tienden a disminuir, esto debido al modelo de demanda utilizado.

En relación con el modelo de tarifas residenciales asumiendo que la mayor recarga de vehículos se hará en hogares, existe una posibilidad de incentivo para los consumidores. Esta opción se basa en la segmentación de periodos de consumo de manera que se promueva la recarga en la noche (de 20:00 a 6:00), lo cual permitiría la normalización de la curva de demanda del Sistema Eléctrico Nacional (SEN). Actualmente, solo la Compañía Nacional de Fuerza y Luz cuenta con el tipo de tarifa residencial horaria (T-REH), la cual se basa en la segmentación de la tarifa en tres periodos: pico, valle y nocturno. Esta tarifa se ofrece a usuarios con consumos mayores a 200 kWh por mes (Compañía Nacional de Fuerza y Luz, 2020). Según Utgård (2017) el 39 \% de clientes residenciales puede acceder a ella, pero solo el $2 \%$ la están apro-

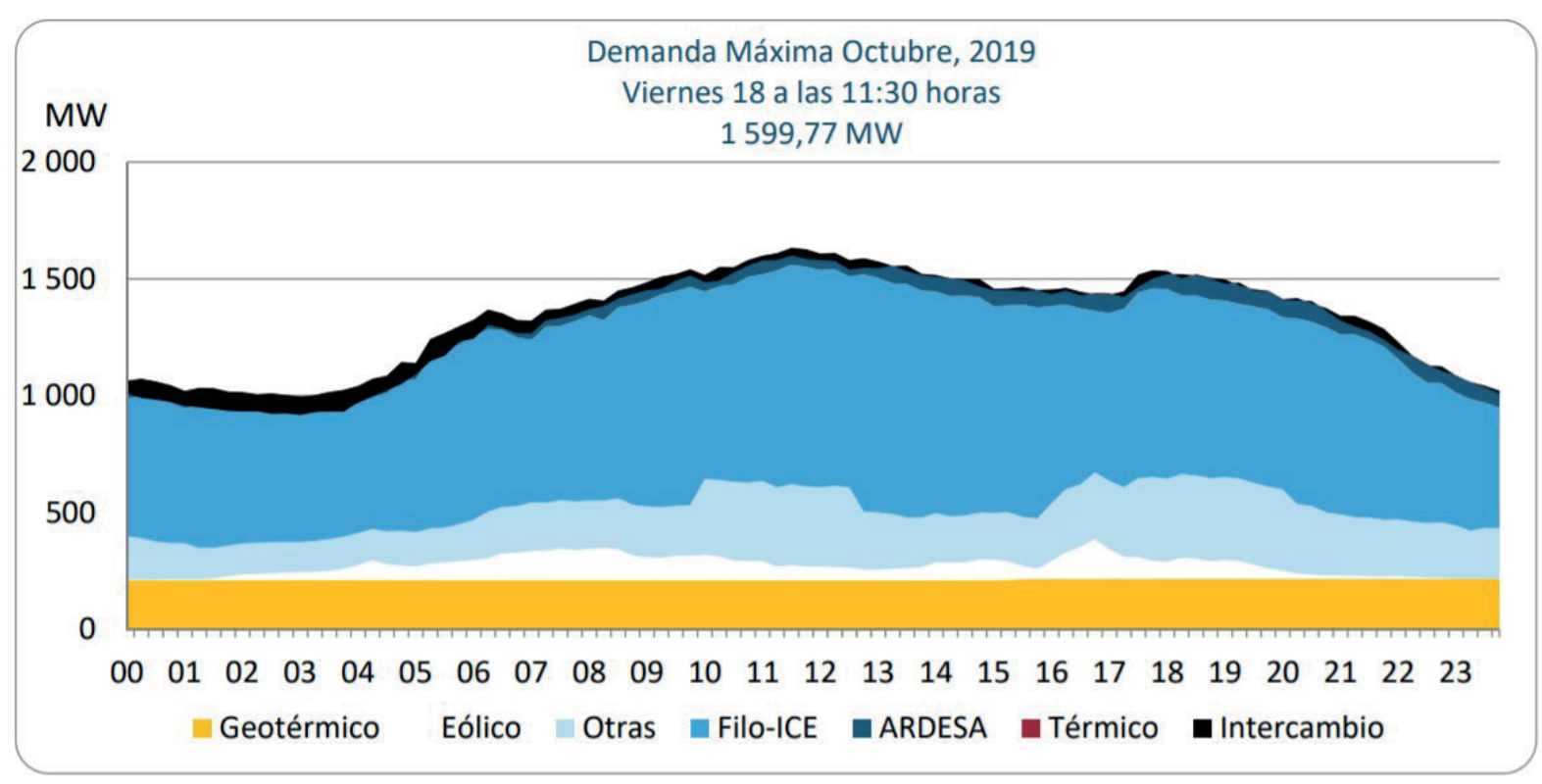

Figura 4. Curva de demanda del SEN para el mes de octubre del 2019. Fuente: curva obtenida del Informe mensual de octubre (CENCE, 2019). 
vechando. Estas oportunidades también se contemplan en el Plan Nacional de Transporte Eléctrico 2018-2030.

\section{Efectos sobre el Sistema Eléctrico Nacional}

Como se mencionó anteriormente, el SEN tiene una generación mediante fuentes renovables del $99 \%$. Normalmente, el comportamiento de la demanda determinada por el perfil de consumo tiene dos picos máximos, donde se ha llegado a valores de $1715 \mathrm{MW}$ como fue el caso de febrero del 2019 (Centro Nacional de Control de Energía, 2019). Para octubre del 2019, el comportamiento se muestra en la figura 4, presentó un máximo de 1,6 GW demandados. (figura 4)

El SEN tiene una potencia instalada de generación aproximada de 3,6 GW, es decir, más de dos veces la potencia diaria demandada normalmente (entre $1,6 \mathrm{GW}$ y $1,7 \mathrm{GW}$ ). Esto quiere decir que existe un gran margen para el aprovechamiento de centrales que operan con un bajo factor de planta. Entonces, ¿cómo afecta el ingreso de vehículos eléctricos al SEN? Depende de varios factores y uno de los más importantes es la hora en la que se recarguen. Lo ideal es evitar que estas nuevas cargas se utilicen en periodos de mayor demanda y para ello existen mecanismos de gestión de demanda que permiten optimizar el uso de la red (Ministerio de Ambiente y Energía, 2019). En Costa Rica los hábitos de conexión de vehículos eléctricos no se conocen, aunque según el Ministerio de Ambiente y Energía (2019) y Utgård (2017) la mayor parte de los propietarios de vehículos eléctricos prefiere cargarlo en la casa durante la noche. Este comportamiento es el más positivo, ya que no afectaría en gran medida la demanda de periodos punta y se aprovecharían el periodo nocturno para la recarga. También cabe la posibilidad de conectarse en otros periodos a las electrolineras o estaciones de carga en carretera, ciudad y otras que deberán considerarse como espacios de emergencias o seguros para eventualidades, puesto que se instalan para permitir el tránsito de viajes largos.

Según Quirós y Blanco (2019) para 200000 vehículos reemplazados por eléctricos en la flotilla costarricense, el SEN no se vería afectado en cuanto a demanda e inclusive se mantendría la generación renovable. Lo cual es indispensable si se quiere disminuir la huella de carbono al no requerir del recurso térmico de respaldo eléctrico. Por el momento, no se considera una gran modificación del SEN, pero, no hay estudios donde se utilicen simulaciones sobre el comportamiento de la demanda al sus- tituir la mayor parte de la flotilla vehicular al menos lo que corresponde a transporte privado (900 000 vehículos aproximadamente).

Cabe resaltar que el Instituto Costarricense de Electricidad en su Plan de Expansión de Generación 2018-2034 proyecta un aumento de capacidad instalada constituido principalmente por geotérmico, eólico y solar, prescindiendo de grandes proyectos hídricos como lo venía planeando en los años anteriores. Este cambio de recursos se debe a la reducción en demanda según lo indican en el informe (Instituto Costarricense de Electricidad, 2019). La capacidad instalada alcanzaría 4076 MW para el 2034, con un crecimiento de $14 \%$ para todo el período 2018-2034. Por lo que el futuro del SEN debe analizarse con la posición de las empresas suministradoras junto a otras tecnologías venideras y que están en desarrollo como las redes inteligentes, Microgrids o Smart Grids de la mano con la generación distribuida.

\section{Integración de los vehículos eléctricos con las redes inteligentes}

La gestión de energía evolucionará a sistemas inteligentes, llamados Smart Grids, en los cuales el flujo de energía eléctrica e información viaja de forma bidireccional. Esto se lograría con la aplicación de sistemas de tecnologías de la información, automatización, Internet de las cosas, inteligencia artificial, Big Data, entre otros. El avance de la generación distribuida, aunado a las mejoras de la tecnología en cuanto a interconexión y recopilación de datos, y la investigación emergente de la aplicación de redes inteligentes, ha causado una integración a un menor nivel, en sistemas llamados microgrids.

Con la electrificación del transporte y su integración en la red, es necesario considerar las fuentes de energía que funcionen de forma aislada que vienen a cambiar la forma en la que comúnmente se dirige energía eléctrica. Los vehículos eléctricos pasan a ser parte del sistema eléctrico y eventualmente podrían funcionar como almacenadores de energía. Según el concepto de Vehicle to grid (V2G), los vehículos eléctricos no solo consumirán energía del sistema, sino que la inyectarán y estabilizarán cuando el sistema lo requiera. Costa Rica posee un sistema eléctrico alimentado por fuentes renovables y que poco a poco está mutando a sistemas de generación locales mediante generación distribuida. Los sistemas de fuentes renovables se caracterizan por ser inestables, ya sea por condiciones climáticas u otros factores. El futuro proyectado por muchos 
investigadores apunta a que los autos también participarán de la regulación de las Smart Grids, para ayudar a la regulación de la tensión y la frecuencia (Tirado y Rivera, 2017).

Según EY (2019), los vehículos eléctricos crearán un "punto de inflexión" que cambiará el juego en la evolución del sistema energético, por lo que requerirá una inversión significativa en nueva infraestructura. Esta empresa investiga sobre el futuro energético y en los resultados, Costa Rica se encuentra en un punto de inflexión cerca del 2025, donde los autos eléctricos serán iguales o superiores en rendimiento e iguales o inferiores en costo comparados a los de combustión interna. (figura 5)

En la figura 5, además, aparecen otros dos puntos de inflexión, el T1 corresponde al de la energía solar en 2032, donde la rentabilidad aumentará en sistemas distribuidos con almacenamiento y será más barato que comprarle energía a una compañía distribuidora; el otro punto T3 se establece para 2046 donde la energía solar obtenida a través de paneles fotovoltaicos y el almacenamiento de esta mediante baterías será más barata que la transmisión y distribución de energía. Con estas proyecciones sobre la electricidad se podrían solidificar los esfuerzos de los gobiernos por incentivar la electrificación del transporte.
El panorama indica que habrá ganadores y perdedores, las Utilities deben adaptarse al comercio futuro donde la energía será intercambiable, inclusive entre vecinos de una misma microrred.

\section{Inteligencia artificial, conducción autónoma y carreteras}

Actualmente, se utiliza inteligencia artificial casi a diario en los teléfonos móviles, sin embargo, los VE lo utilizan a un nivel superior para interactuar con el entorno físico. Muchos de los fabricantes de VE lo están investigando, pero hoy, el que lleva el estandarte es Tesla Motors, que ha integrado sensores, radares y cámaras con software para crear un asistente de manejo: el autopilot que ayuda al conductor a trasladarse, principalmente, en autopistas sin tocar el volante ni pedales (conducción de nivel 3). El objetivo de esta tecnología es convertirse en conducción autónoma, de manera que el VE sea capaz de entender los complejos entornos de carreteras y responder a eventualidades que puedan ocurrir.

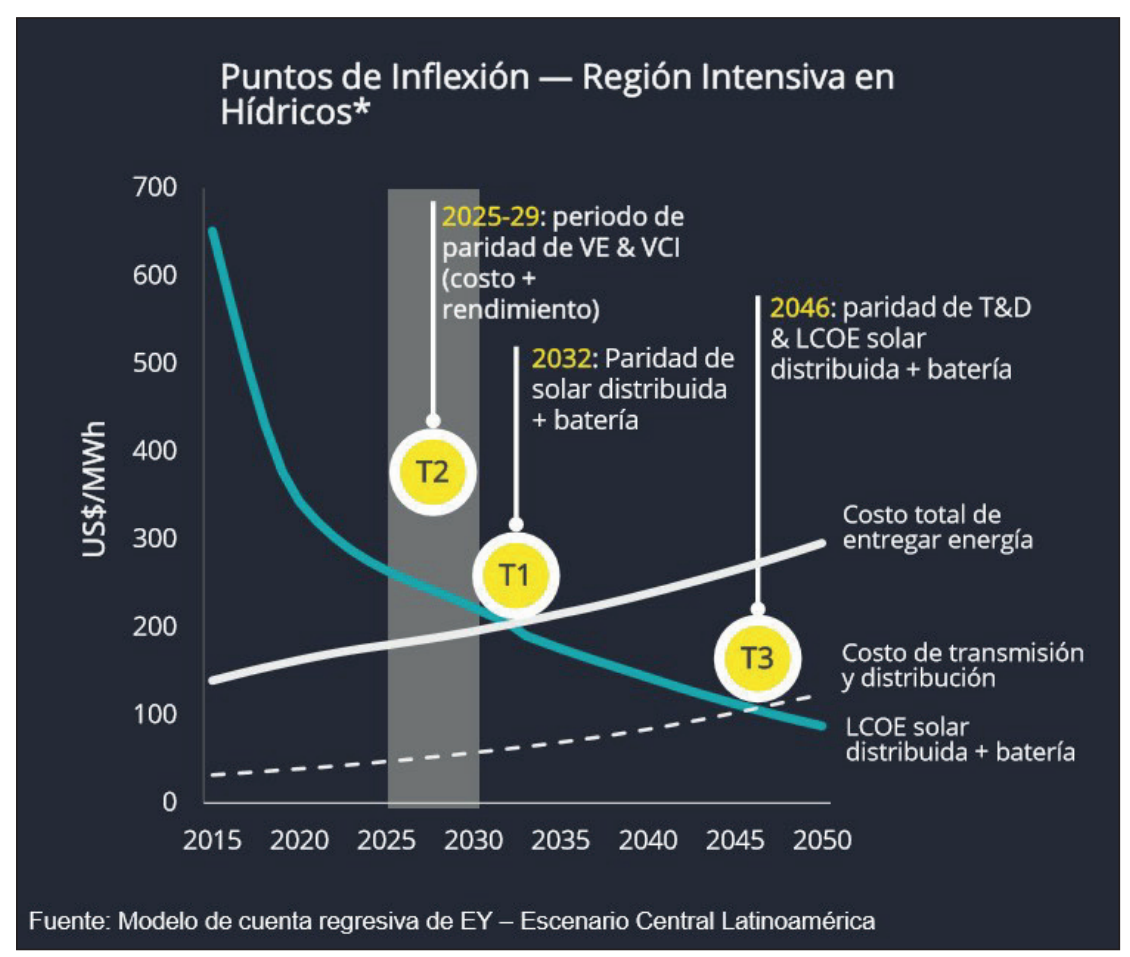

Figura 5. Puntos de inflexión según la región: Latinoamérica, región hídrica. Fuente: Caso de estudio (EY, 2019). 


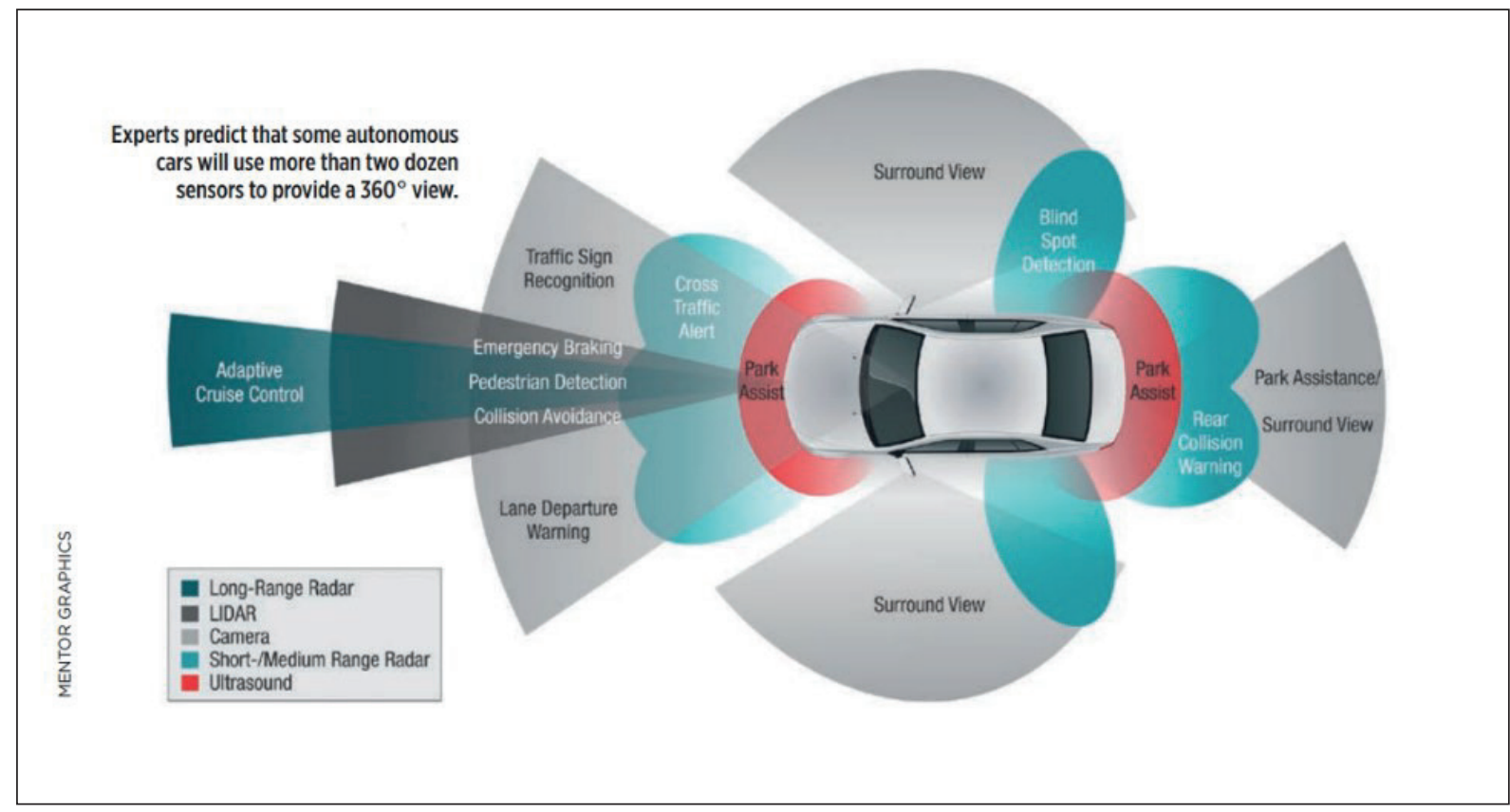

Figura 6. Sensores, cámaras y radares incorporados en vehículos eléctricos inteligentes que permiten la conducción autónoma. Fuente: Costlow, 2019

La tecnología de autonomía se ayuda de carreteras bien marcadas, con señalización completa. En Costa Rica las carreteras en algunos casos no cumplen con los requisitos para aplicarla, aunque probablemente en el futuro el entrenamiento de las redes neuronales que controlan las decisiones en operación de los VE superará hasta cierto punto las limitaciones espaciales. No todos los sucesos pueden ser considerados puesto que, existirá siempre un mínimo de error, producido por la "cola larga" de eventos en carretera.

La conducción autónoma se divide en cinco niveles: el 0 (sin automatización) corresponde al actual, los conductores se encargan del control total del vehículo, acelerar y desacelerar cuando se debe y se controlan las variables del entorno. En el nivel 1 (asistencia de conducción), el vehículo puede ayudar con algunas funciones, pero el conductor aún maneja toda la aceleración, frenado y monitoreo del entorno. En el nivel 2 (automatización parcial), según Harner (2017), la mayoría de los fabricantes de automóviles están desarrollando vehículos en este nivel, donde el vehículo puede ayudar con las funciones de dirección o aceleración y permitir al conductor desconectarse de algunas de sus tareas. El conductor siempre debe estar listo para tomar el control del vehículo y seguir siendo responsable de la mayoría de las funciones críticas de seguridad y de todo el monitoreo del medio ambiente. El nivel 3 (automatización condicional) se caracteriza porque el vehículo controla toda la monitorización del entorno. En los niveles 4 y 5, el vehículo es capaz de conducir, frenar, acelerar, monitorear el vehículo y la carretera, así como responder a eventos, determinar cuándo cambiar de carril, girar y usar las señales. La diferencia es que en el quinto nivel de conducción autónoma no se requiere absolutamente ninguna atención humana.

\section{Carpooling}

Además, la Inteligencia Artificial trae consigo el concepto de autos compartidos, donde varios usuarios podrán utilizar un auto eléctrico inteligente y autónomo si las condiciones así lo permiten. Por lo que se han desarrollado en el país aplicaciones que promueven el compartir espacios en autos cuando las personas se dirigen a un mismo destino. Este tipo de servicio trata de intentar aprovechar mejor el tiempo y la energía invertidas en transporte. En el futuro se proyecta una utilización mayor en donde no se requieran conductores, sino solo pasajeros que le indican a un VE donde quieren ir con respecto a una ruta coincidente con la de los demás usuarios. Si esto se llegara a desarrollar sólidamente, el consumo y, por tanto, el impacto per cápita disminuiría considerablemente. Sin embargo, se requieren años de transición, cambios de mentalidad y cultura de la población costarricense para lograr aprovechar este modelo. 


\section{Vehículos eléctricos de hidrógeno}

Los vehículos eléctricos con baterías de almacenamiento no es la única alternativa existente al problema energético de transporte. Se está invirtiendo fuertemente en investigación de vehículos con celda de hidrógeno y en general el hidrógeno como vector energético. En cuanto a los vehículos de hidrógeno constan de una pila capaz de realizar el proceso inverso a la electrólisis, liberando energía eléctrica al unir moléculas de oxígeno e hidrógeno, siendo el único residuo el vapor de agua y calor. La energía eléctrica alimentará al motor eléctrico incorporado en estos equipos (BMW, 2020). Estas celdas o baterías funcionan con alta eficiencia, la marca Toyota la está implementando en sus vehículos, como por ejemplo el Mirai, el primero en producirse masivamente según esta tecnología. En Costa Rica el Dr. Franklin Chang impulsa el desarrollo del hidrógeno como almacenamiento energético. No obstante, la producción de hidrógeno y presiones de almacenamiento deben investigarse más para alcanzar mejores eficiencias.

\section{Conclusiones}

Tal como se ha analizado, Costa Rica tiene una dependencia de hidrocarburos en cuanto a transporte, sector de mayor consumo, pero también es un país con un potencial eléctrico para la reconversión de la flotilla a vehículos eléctricos. Sus recursos energéticos, el sistema eléctrico, la legislación en cuanto al transporte eléctrico y proyectos energéticos abren paso a una transición tecnológica. La construcción de cargadores rápidos de nivel 3 en todo el territorio nacional es necesaria para suministrar la autonomía requerida para cualquier viaje planeado por la población costarricense. Actualmente, en el país es 3 veces más económica la operación de un VE que uno de combustión interna.

Asimismo, la afectación al SEN es soportada por la sobreinstalación de generación, al menos en los primeros 200000 vehículos eléctricos de la flotilla según los datos analizados. No obstante, deben realizarse estudios y simulaciones para conocer los efectos finales sobre el sistema eléctrico con el cambio de la totalidad del parque vehicular considerando las proyecciones del Plan de Expansión del Instituto Costarricense de Electricidad y el aumento de generación distribuida. El cambio completo no solo generaría mayor demanda eléctrica, sino que hay que estudiar los beneficios de compensación que podrían tener los autos eléctricos conectados en redes inteligentes en el futuro, donde amortiguarían las variaciones de tensión y frecuencias según el concepto de V2G.

La disrupción de los VE en Latinoamérica tiene un punto de inflexión cerca de los seis años para países como Costa Rica, donde se explotan mayoritariamente recursos hídricos en generación, según los datos estudiados. La transición trae consigo una serie de tecnologías disruptivas que marcarán la era de la interconexión inteligente.

En un futuro se disfrutarán de los beneficios de la conducción autónoma, lo que provocará un cambio de cultura donde las personas utilicen un carro que no les pertenece para transportarse. La mayor parte del tiempo los vehículos propios pasan estacionados, una utilización grupal reduciría tiempos de tráfico y el consumo energético per cápita. Otra opción considerada para el futuro de la movilidad costarricense es el hidrógeno, la cual coexistirá junto a los VE de batería hasta encontrar el recurso más eficiente.

\section{Referencias}

Arrieta, E. (14 de noviembre de 2018). Costa Rica es el tercer país con mayor densidad vehicular de Latinoamérica. La República. https://bit.ly/2sdvuuk

BMW. (17 de marzo de 2020). Coches de hidrógeno: todo lo que debes saber. Obtenido de BMW: https://bit.ly/3cKZYW7

Brenes Brenes, R. (2016). Ubicación y dimensionamiento de estaciones de recarga rápida de vehículos eléctricos en redes de distribución utilizando información GIS. Proyecto de grado, Universidad de Costa Rica, Escuela de Ingeniería Eléctrica, Ciudad Universitaria Rodrigo Facio. https://bit. ly/2ASILxQ

Centro Nacional de Control de Energía. (octubre de 2019). Informe mensual Octubre 2019. CENCE: https:// apps.grupoice.com/CenceWeb/CenceMain.jsf

Centro Nacional de Control de Energía. (abril de 2020). Informe Mensual Abril 2020. CENCE: https:// apps.grupoice.com/CenceWeb/CenceMain.jsf

Compañía Nacional de Fuerza y Luz. (24 de abril de 2020). Tarifa Residencial Horaria T-REH. CNFL: https://bit.ly/3dL8LbS 
Costlow, T. (13 de febrero de 2019). Fusing Sensors for the Automated Driving Future. Sae Mobilus: https://bit.ly/2PtTLnW

Crabtree, G. (25 de octubre de 2019). The coming electric vehicle transformation. Science Mag: https:// science.sciencemag.org/content/366/6464/422

Electromaps. (10 de diciembre de 2019). Puntos de recarga Costa Rica. https://www.electromaps.com/ puntos-de-recarga/costa-rica

Enel X. (20 de abril de 2019). The Different EV Charging Connector Types. Enel X: https://bit.ly/3he2esC

EY. (2019). Cuando los clientes se salgan de la red, ¿quedarán a oscuras las empresas de energía. EY: https://bit.ly/2t1qmK8

Faraj, M., \& Basir, O. (2016). Range anxiety reduction in battery-powered vehicles. 2016 IEEE Transportation Electrification Conference and Expo (ITEC), 1-6. doi:10.1109/ITEC.2016.7520190

Gallardo, L., Angulo, J., Bejarano, R., Fuentes, D., Ruiz, L., Martínez, J., \& Quirós, J. (2019). Strategic Location of EV Fast Charging Stations: The Real Case of Costa Rica. 2019 IEEE PES Innovative Smart Grid Technologies Conference - Latin America (ISGT Latin America). https://ieeexplore.ieee. org/document/8895284

Harner, I. (23 de octubre de 2017). The 5 Autonomous Driving Levels Explained IoT for All: https:// www.iotforall.com/5-autonomous-driving-levels-explained/

Herrera, W. (24 de septiembre de 2019). Costa Rica es el tercer país con más autos eléctricos en Latinoamérica. La República.

Instituto Costarricense de Electricidad. (mayo de 2019). Plan de Expansión de la Generación Eléctrica. Grupo ICE: https://bit.ly/2ASCHEB
Ministerio del Ambiente y Energía. (2019). Plan Nacional de Transporte Eléctrico. San José. https://sepse.go.cr/documentos/PlanTranspElect.pdf

Plugshare. (9 de diciembre de 2019). Agencia ICE Puntarenas. https://www.plugshare.com/location/175349

Presidencia de Repúplica de Costa Rica. (2 de abril de 2019). Gobierno anuncia Red Nacional de Carga Rápida para vehículos eléctricos en todo el país. https://bit.ly/36p28Io

Programa estado de la nación. (2019). Informe estado nación. PEN: https://estadonacion.or.cr/informe/

Quirós, J., \& Blanco, J. [Canal UCR]. (10 de junio de 2019). Especiales UCR: Autos eléctricos ¿Para quién? [Archivo de video] https://youtu.be/xtR$6 \mathrm{~Bq} 855 \mathrm{rw}$

Secretaría Planificación Subsector Energía. (2019). Balance energético nacional 2018. SEPSE: https:// sepse.go.cr/ciena/balances-energeticos/

Tirado, S., \& Rivera, S. (2017). Vehículos eléctricos como controladores de frecuencia en una microred. Journal de Ciencia e Ingeniería, 10(1), 6-17. https://biblioteca.utn.ac.cr/

Umaña, J. (7 de septiembre de 2017). Autos eléctricos: condiciones de Costa Rica auguran un futuro prometedor. Hoy en el TEC: https://bit.ly/2E4Fadt

Utgård, B. (abril de 2017). Esencialmente eléctrica: Cómo puede abanderar Costa Rica la movilidad eléctrica? Costa Rica Limpia: https://bit.ly/2rxTjgk

Utgård, B., Bermúdez, E., \& Araya, M. (noviembre de 2016). Mitos comunes ticos sobre el transporte eléctrico. Costa Rica Limpia: https://bit.ly/38r$\mathrm{d} 0 \mathrm{r} 2$ 